\title{
New Method for Encapsulation of Oregano Essential Oil into Carbon Nanotubes
}

\author{
Mariana PRODANA ${ }^{1}$, Madalina Georgiana ALBU ${ }^{2}$, Durmus Alpaslan \\ KAYA $^{3}$, Andrada NEGRU ${ }^{1,4}$, Dionezie BOJIN ${ }^{1}$, Marius \\ ENACHESCU ${ }^{1 *}$ \\ ${ }^{1}$ Center for Surface Science and Nanotechnology, \\ University "Politehnica" of Bucharest, 060042, Bucharest, Romania \\ ${ }^{2}$ Collagen Department, INCDTP - Division Leather and Footwear Research \\ Institute, 031215, Bucharest, Romania \\ ${ }^{3}$ Department of Field Crops, Faculty of Agriculture, Mustafa Kemal \\ University, 31030, Antakya-Hatay, Turkey \\ ${ }^{4}$ University of Pitesti, 110040, Pitesti, Romania \\ *e-mail: marius.enachescu@upb.ro
}

\begin{abstract}
In this paper it is proposed a new method of encapsulation of oregano essential oil (EO) into carbon nanotubes, singlewalled (SWCNTs) and multiwalled (MWCNTs). Multiwalled carbon nanotubes were functionalized by oxidation methods to obtain carboxilated carbon nanotubes MWCNT-COOH and aminated carbon nanotubes MWCNT- $\mathrm{NH}_{2}$. The effect of encapsulating matrix on protection and delivery of oregano essential oil was studied. We will refer to preparation by encapsulation of carbon nanotubes with oregano essential oil in order to improve characteristics that can be used in biomedical applications. EO was encapsulated in carbon nanotubes by physical immersion and the samples were ultrasonated for 2 hours at $37^{\circ} \mathrm{C}$. After the treatment of carbon nanotubes with oregano essential oil, these varieties of materials were inserted into a collagen gel and lyophilized in order to obtain collagen matrices. The structure of the new functionalized carbon nanotubes immersed in a collagen matrix was identified using infrared spectroscopy (FTIR analysis) and morphological features were studied by transmission electron microscopy (TEM) and scanning electron microscopy (SEM).

Keywords: carbon nanotubes, oregano oil, TEM, FTIR.
\end{abstract}

\section{Introduction}

Oregano is an aromatic plant from Mediterranean countries widely used as food ingredient due to its specific flavour. Moreover, oregano oil is a strong antioxidant [1] with very good antimicrobial properties being a good alternative for food preservative. The use of EO as health promoting substance is directly related with some components of oregano such as carvacrol and thymol.

Microencapsulation is the technique that allows sensitive ingredients to be entrapped in every kind of homogenous or heterogeneous matrix but the choice of material is an important key for the success of encapsulation process.

Carbon nanotubes (CNTs) [2,3] were widely used because posses exceptional electrical, thermal and mechanical properties and is not being degraded in human body; they can be used to deliver small organic drug molecules into the diseased cells thus preventing normal tissue damages. Due to the chemical oxidation CNTs can be very easy functionalized and they can be used as targeting materials in combination with cisplatin (potent anticancer agent). Single wall carbon nanotubes (SWCNTs) potential can be 
exploited by uploading the form of antineoplastic compound shell at the external tube by a covalent bond. The drug was then released as active form as the reduction reaction in acidic endosomes and lysosomes.

\section{Experimental methods}

\subsection{Materials and Reagents}

High-purity SWCNTs were purchased from Sigma Aldrich. These SWCNTs were produced by a chemical vapor deposition process yielding particle external diameters of less than $2 \mathrm{~nm}$ with lengths ranging from 0.5 to 40 microns and a purity $>90 \%$.

Multiwall Carbon Nanotubes (MWCNTs) were purchased from Sigma Aldrich having more than $90 \%$ carbon basis and D x L 10$15 \mathrm{~nm} \times 0.1-10 \mu \mathrm{m}$, produced by Catalytic Chemical Vapor Deposition (CCVD). Oxidation was made using a mixture of 98\% sulfuric acid (Merck).

\subsection{Equipment}

FTIR spectra of functionalized MWCNTs were registered on a Perkin Elmer, Spectrum 100 equipment in $400 \div 4500 \mathrm{~cm}^{-1}$ range with $4 \mathrm{~cm}^{-1}$ resolution and 32 scans.

Nano-sized particles were investigated using TEM analysis with a microscope Philips EM-410, 60kV and by SEM using a SU8230 microscope.

\subsection{Procedure}

Multiwalled carbon nanotubes were functionalized with carboxil $-\mathrm{COOH}$, cisplatin -CDDP and amino $-\mathrm{NH}_{2}$ groups.

\section{Functionalization of MWCNTs with - COOH groups:}

MWCNTs $(2.0 \mathrm{~g})$ were dispersed in $98 \%$ concentrated sulphuric acid under ultrasonication at $50^{\circ} \mathrm{C}$ for $6 \mathrm{~h}$ to produce oxidized carbon nanotubes (MWCNT$\mathrm{COOH})$ [4]. The samples were washed with ultrapure water and dried at $50{ }^{\circ} \mathrm{C}$ for $12 \mathrm{~h}$.

\section{Obtaining of MWCNTs with -CDDP}

CDDP (5 mg) was added to MWCNT$\mathrm{COOH}$ solutions in $1 \mathrm{ml}$ saline solution.
They were ultrasonicated for 48 hours at $50^{\circ} \mathrm{C}$ and filtered.

\section{Functionalization of MWCNT- $\mathrm{NH}_{2}$}

Dried MWCNT-COOH (0.1 mg) was reacted with excess $\mathrm{SOCl}_{2}(25 \mathrm{~mL})$ at room temperature for 30 minutes. The residual $\mathrm{SOCl}_{2}$ was removed by washing with tetrahydrofuran THF and filtered with ultrapure water.

The MWCNTs were dried for 20 minutes at the room temperature.

The new functionalized nanotubes MWCNT-SOCl 2 (10 mg) are added in etilendiamine in excess for 10 hours at the room temperature. The mixture was washed with THF and filtered. The nanotubes were dried at $80^{\circ} \mathrm{C} / 10$ hours [5].

\section{Encapsulation of oregano essential oil}

The encapsulation of oregano essential oil consists of adding $5 \mathrm{ml}$ of EO into samples that contain carbon nanotubes (functionalized and nonfunctionalized, single or multiwall), under ultrasonication for 2 hours at $37^{\circ} \mathrm{C}$.

We will refer to 5 types of samples such as: -sample 1: SWCNTs+EO; -sample 2: MWCNTs+EO; -sample 3: MWCNT-COOH+EO; -sample 4: MWCNT-CDDP+EO; -sample 5: MWCNT-NH+ EO.

After that encapsulation the samples was characterized by TEM and FTIR.

Type I collagen of bovine origin was extracted by the currently used technology as previously described [6]. The collagen (Coll) was obtained as gel in native form with fibrillary structure with an initial concentration of $2.11 \%, \mathrm{pH} 2.5$ and free of fat and ash. Glutaraldehyde (GA) was supplied by Sigma-Aldrich (Germany) and sodium hydroxide from Merck (Germany). All the chemicals used in this work were of analytical grade and the water was distilled. The obtained functionalized carbon nanotubes encapsulated with oregano essential were added to collagen gel with ratio 1:10 (reported to dry collagen). The composite gels based on collagen as such 
(reference sample) and collagen with CNTs were adjusted at physiological $\mathrm{pH}$ (7.2 7.4 ), $1.2 \%$ collagen (dry substance) and then cross - linked with $0.5 \%$ GA. The cross-linked gels were frozen at $-40^{\circ} \mathrm{C}$ for 12 hours and then freeze-dried according to the method previously described [7] using the Christ Model Delta 2-24 LSC freezedryer (Germany). 3D spongious composites were obtained and characterized as by SEM.

\section{Results and discussions}

\subsection{TEM analysis:}

Nanostructured composite morphologies are investigated using a transmission electron microscope EM-410, 60kV. The morphologies obtained for every sample are presented in figures 1 .

For the first sample (fig. 1a) the morphology is specified for single-wall carbon nanotubes. The structure is uniform and tubular distributed on the surface of a cooper grid. The average diameter of SWCNTs nanotubes was measured with Image J program and the value is almost $1.2 \pm 0.2 \mathrm{~nm}$. From place to place, it is visible on the TEM micrographies, the encapsulation of oregano oil into SWCNTs.

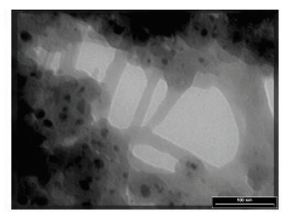

a. SWCNTs

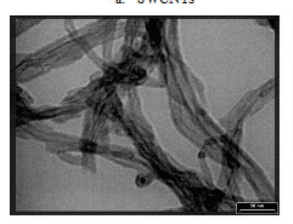

c. MWCNT-COOH

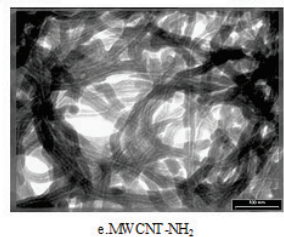

e.MWCNT-NH

Figure 1. TEM micrographies for samples encapsulated with oregano essential oil
The encapsulation of EO into MWCNTs is put in evidence in fig. $1 \mathrm{~b}$. The net of nonfunctionalized multiwalled carbon nanotubes is very dense and encapsulation takes place at the ends of carbon nanotubes, at the extremities. The average diameter of these kinds of carbon nanotubes was also measured with ImageJ soft and the value is almost $9 \pm 1 \mathrm{~nm}$. The encapsulation of oregano essential oil it is also very well put in evidence in images $1 \mathrm{c}, \mathrm{d}$ and $\mathrm{e}$.

\subsection{FTIR determinations:}

The samples were analyzed using FTIR and infrared Microscopy Spectral data were recorded by an ATR Perkin-Elmer equipment. In figures 2 are presented the FTIR of super reposed of individual spectra of $1 \div 5$ samples.

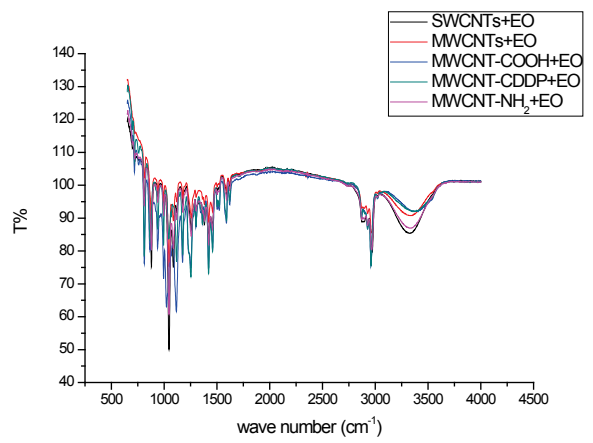

Figure 2. FTIR for samples encapsulated with oregano essential oil

The $-\mathrm{COOH}$ and $-\mathrm{NH}_{2}$ functionalization is put in evidence by FTIR measurements.

FTIR has been used to map the chemical distribution of carbon nanotubes SWCNTs, MWCNTs, acid treated MWCNT-COOH, MWCNT-CDDP and MWCNT-NH 2 .

FTIR spectra for the super reposed of $1 \div 5$ samples show a broad peak at $3406 \mathrm{~cm}^{-1}$, which refers to the $\mathrm{O}-\mathrm{H}$ stretch of the hydroxyl group which can be ascribed to the oscillation of carboxyl groups.

Carboxyl groups on the surfaces of MWCNTs could be due to the partial oxidation of the surfaces of MWCNTs during purification by the manufacturer, $1626 \mathrm{~cm}^{-1}$ and is associated with the stretch 
mode of carboxylic groups $(\mathrm{O}=\mathrm{C}-\mathrm{OH}$ and $\mathrm{C}-\mathrm{OH}$ ), as observed in the IR spectrum of MWCNT-COOH.

Carboxylic groups are formed due to the oxidation of carbon atoms on the surfaces of the MWCNTs by sulphuric acid. The peak at $1878 \mathrm{~cm}^{-1}$ can be associated with the $\mathrm{O}-\mathrm{H}$ stretch from strongly hydrogenbonded $-\mathrm{COOH}$. The peak at $1592 \mathrm{~cm}^{-1}$ is related to the carboxylate anion stretch mode. The peak at $1629 \mathrm{~cm}^{-1}$ can be associated with the stretching of the carbon nanotube backbone. The peaks at around 2870 and $2927 \mathrm{~cm}^{-1}$ correspond to the $\mathrm{H}-\mathrm{C}$ stretch modes of $\mathrm{H}-\mathrm{C}=\mathrm{O}$ in the carboxyl group.

For MWCNT-CDDP present a peak at around $868 \mathrm{~cm}^{-1}$ specific for -CDDP.

For MWCNT-NH$H_{2}$ the bands at 2929 and $2873 \mathrm{~cm}^{-1}$ represent asymmetrical and symmetrical stretching of $\mathrm{CH}_{2}$ groups. The $\mathrm{C}-\mathrm{N}$ stretching vibration and the scissoring in-plane $\mathrm{N}-\mathrm{H}$ distortion of free primary amine group are observed at $1047 \mathrm{~cm}^{-1}$ and $1622 \mathrm{~cm}^{-1}$, respectively. The broad band at $3355 \mathrm{~cm}^{-1}$ is attributed to the $\mathrm{NH}_{2}$ stretching.

We supposed that any other peaks presented in individual spectrum of each sample are specific for oregano essential oil encapsulation.

The addition of EO resulted in a markedly increased in intensity of the $\mathrm{CH}$ stretching peak at 2867-2955 $\mathrm{cm}^{-1}$, indicating an increase in the content of ester groups, which might come from EO molecules. The peak at $2961 \mathrm{~cm}^{-1}$ is due to the presence of $-\mathrm{CH}$ stretching, the peak at $1594 \mathrm{~cm}^{-1}$ represent the N-H bending, at $1459 \mathrm{~cm}^{-1}$ is $\mathrm{CH}_{2}$ bending, at $1255 \mathrm{~cm}^{-1}$ and $1121 \mathrm{~cm}^{-1}$ is put in evidence the $-\mathrm{C}-\mathrm{O}-\mathrm{C}$ - stretching and $936 \mathrm{~cm}^{-1}$ is specific for $\mathrm{C}-\mathrm{H}$ bending.

Figure 3 present some literature results for FTIR spectra [8].

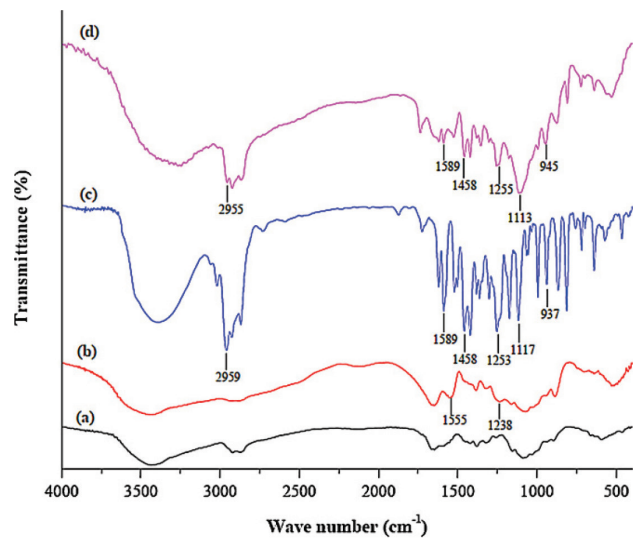

Figure 3. Literature: (c) spectrum is for oregano oil [8] and (d) is for oregano oil+chitosan seems similar with ours spectra

\subsection{SEM morphology}

The morphological aspects of the samples surface, with CNTs and EO into the collagen matrix and the pore interconnectivity were determined by Scanning Electron Microscopy, using a Hitachi SU8230 microscope at $5.0 \mathrm{kV}$

The morphology is presented in figure 4 .

The SEM investigation offer information about the morphology and distribution of the particle to/at the collagen matrix.

In figure $4 \mathrm{a}$ is presented the morphology of the composite that involves SWCNTs, oregano oil and collagen.

The figure $4 \mathrm{~b}$ presents the morphology of the composite made by MWCNTs, oregano oil and collagen.

Both pictures show some similarities. The structure is a porous one.

The morphological results by SEM analysis revealed a differentiated homogeneity and pore interconnectivity in our composites.

The walls of the collagen network appear to be thicker for the composite containing single-wall carbon nanotubes than for the composite that contain multiwall carbon nanotubes. 


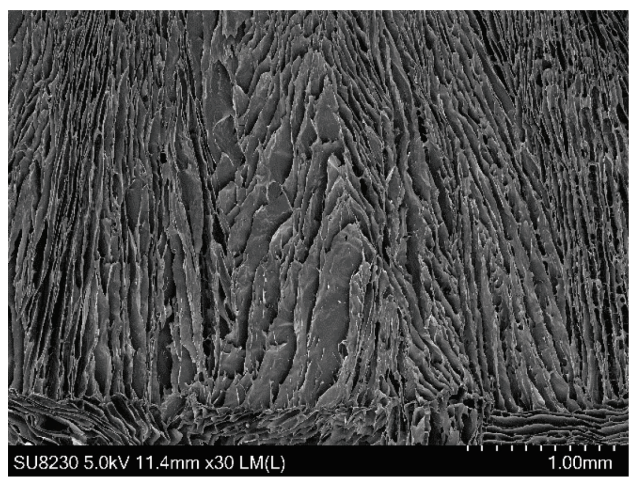

a)

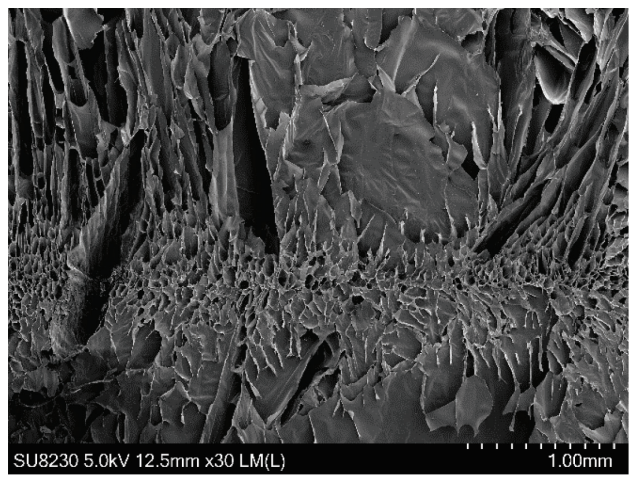

b)

Figure 4. SEM image of the collagen based matrix: a) with SWCNTs; b) with MWCNTs

\section{Conclusions}

Results show that both kind of carbon nanotubes, single or multiwalled were encapsulating quite well the oregano essential oil. The SEM images of the composite revealed a high porous structure, common in all samples. This kinds of materials encapsulated further in a collagen matrix seems to be very promising for biomedical applications. More tests will be proceed in order to study the biocompatibility of our composites based on collagen matrix.

\section{Acknowledgment}

This work was supported by Romanian Ministry of Education and by Executive Agency for Higher Education, Research, Development and Innovation Funding, under projects PCCA 2- nr. 66/2014 and PCCA 2-nr. 166/2012.

\section{References}

1) S. Beirão da Costa, C. Duarte, A.I. Bourbon, A.C. Pinheiro, A.T. Serra, M.M. Martins, M.I.N. Januário, A.A. Vicente, I. Delgadillo, C. Duarte, M.L. Beirão da Costa, Journal of Food Engineering 110 (2012) 190.

2) S. Iijima, Nature 354 (1991) 56.

3) Z. Zhang, B. Liu, Y.W. Zhang, K.C. Hwang, H. Gao, Carbon 77 (2014) 1040.

4) M. Prodana, D. Ionita, D. Bojin, I. Demetrescu Adv. Sci. Lett. 5 (2012) 1.

5) M. Prodana, D. Ionita, D. Bojin, I. Demetrescu, Journal of Sustainable Energy 3 (2) (2012) 62.

6) M.G. Albu Collagen gels and matrices for biomedical applications, edited by E. Alexei (Lambert Academic Publising, Saarbrücken, Germany 2011)

7) M.G. Albu, M.V. Ghica, Farmacia 63(1) (2015) 20.

8) S.F. Hosseini, M. Zandi, M. Rezaei, F. Farahmandghavi, Carbohydrate Polymers 95 (2013) 50. 